\title{
Diagnosis of Balamuthia mandrillaris Encephalitis by Thymine-Adenine Cloning Using Universal Eukaryotic Primers
}

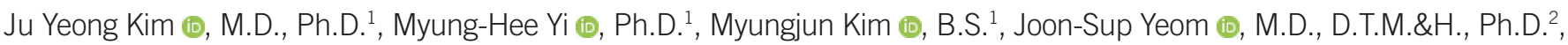

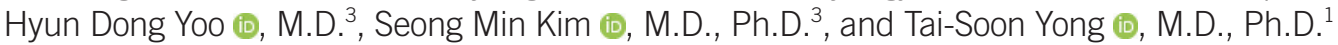

${ }^{1}$ Department of Environmental Medical Biology, Institute of Tropical Medicine, Arthropods of Medical Importance Resource Bank, Yonsei University College of Medicine, Seoul, Korea; ${ }^{2}$ Department of Internal Medicine, Yonsei University College of Medicine, Seoul, Korea; ${ }^{3}$ Department of Neurosurgery, Eulji University Hospital, College of Medicine, Eulji University, Daejeon, Korea

Background: Identifying the causal pathogen of encephalitis remains a clinical challenge. A 50-year-old man without a history of neurological disease was referred to our department for the evaluation of an intracranial lesion observed on brain magnetic resonance imaging (MRI) scans, and the pathology results suggested protozoal infection. We identified the species responsible for encephalitis using thymine-adenine (TA) cloning, suitable for routine clinical practice.

Methods: We extracted DNA from a paraffin-embedded brain biopsy sample and performed TA cloning using two universal eukaryotic primers targeting the V4-5 and V9 regions of the $18 \mathrm{~S}$ rRNA gene. The recombinant plasmids were extracted, and the inserted amplicons were identified by Sanger sequencing and a homology search of sequences in the National Center for Biotechnology Information Basic Local Alignment Search Tool.

Results: The infection was confirmed to be caused by the free-living amoeba Balamuthia mandrillaris. Two of 41 colonies recombinant with $18 S$ V4-5 primers and 35 of 63 colonies recombinant with the $18 \mathrm{~S}$ V9 primer contained $B$. mandrillaris genes; all other colonies contained human genes. Pathogen-specific PCR ruled out Entamoeba histolytica, Naegleria fowleri, Acanthamoeba spp., and Toxoplasma gondii infections.

Conclusions: This is the first report of $B$. mandrillaris-induced encephalitis in Korea based on molecular identification. TA cloning with the 18S rRNA gene is a feasible and affordable diagnostic tool for the detection of infectious agents of unknown etiology.

Key Words: Amoeba, Balamuthia mandrillaris, Encephalitis, TA cloning, 18S rRNA
Received: January 10, 2021

Revision received: March 7, 2021

Accepted: September 14, 2021

\section{Corresponding author:}

Tai-Soon Yong, M.D., Ph.D.

Department of Environmental Medical Biology, Institute of Tropical Medicine, Arthropods of Medical Importance Resource Bank, Yonsei University College of Medicine, 50-1 Yonsei-ro, Seodaemungu, Seoul 03722, Korea

Tel: +82-2-2228-1841

Fax: +82-2-363-8676

E-mail: tsyong212@yuhs.ac

\section{Co-corresponding author:}

Seong Min Kim, M.D., Ph.D.

Department of Neurosurgery, Eulji

University Hospital, College of Medicine,

Eulji University, 95 Dunsanseo-ro, Seo-gu,

Daejeon 35233, Korea

Tel: +82-42-611-3442

Fax: +82-42-611-3444

E-mail: nsksm@eulji.ac.kr

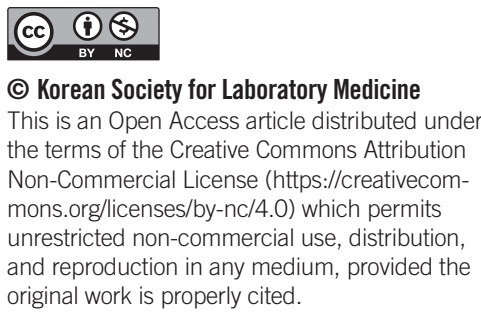

\section{INTRODUCTION}

Identifying the causes of encephalitis can be challenging because various bacterial, viral, fungal, and parasitic infections can man- ifest with overlapping symptoms and signs. Pathogen-specific serological, PCR, and culture-based analyses are expensive, time-consuming, and lack standardization [1]. Recently, metagenomic next-generation sequencing (NGS) has been used to 
identify unusual or novel pathogens, including those causing meningoencephalitis. For example, Leptospira, a spirochaete, and Balamuthia mandrillaris, a free-living amoeba, have been identified in patients with meningoencephalitis by metagenomic NGS [1, 2]. Despite the several advantages of this approach, the detection of unusual protozoan infections by NGS within a reasonable timeframe is not always feasible for routine practice owing to the need for specialized equipment, and the time and expertise required for bioinformatic analyses. As an alternative, thymine-adenine (TA) cloning is more feasible and affordable than NGS. Here, we describe a patient with encephalitis caused by $B$. mandrillaris that was identified using the TA cloning method. To the best of our knowledge, this is the first report on $B$. mandrillaris gene detection in Korea. Cloning was performed using universal 18S rRNA gene primers that have been widely applied in eukaryotic metagenomic studies [3]. We initially identified all protozoal species in the brain sample, because B. mandrillaris infection was unknown in Korea at the time and was not initially considered.

\section{MATERIALS AND METHODS}

Brain biopsy sample from a patient with encephalitis A 50-year-old man without a history of neurological disease was referred to the Department of Neurosurgery, Eulji University Hospital, Daejeon, Korea, for evaluating an intracranial lesion visible on his brain magnetic resonance imaging (MRI) scans. The patient had a three-day history of mild speech disturbance and right-sided hemiparesis after a focal seizure. He did not chew tobacco, drink alcohol, or use drugs and showed no signs of infection, such as fever, leukocytosis, and meningeal irritation. A brain MRI revealed a mass in the cortex of the left parietal lobe
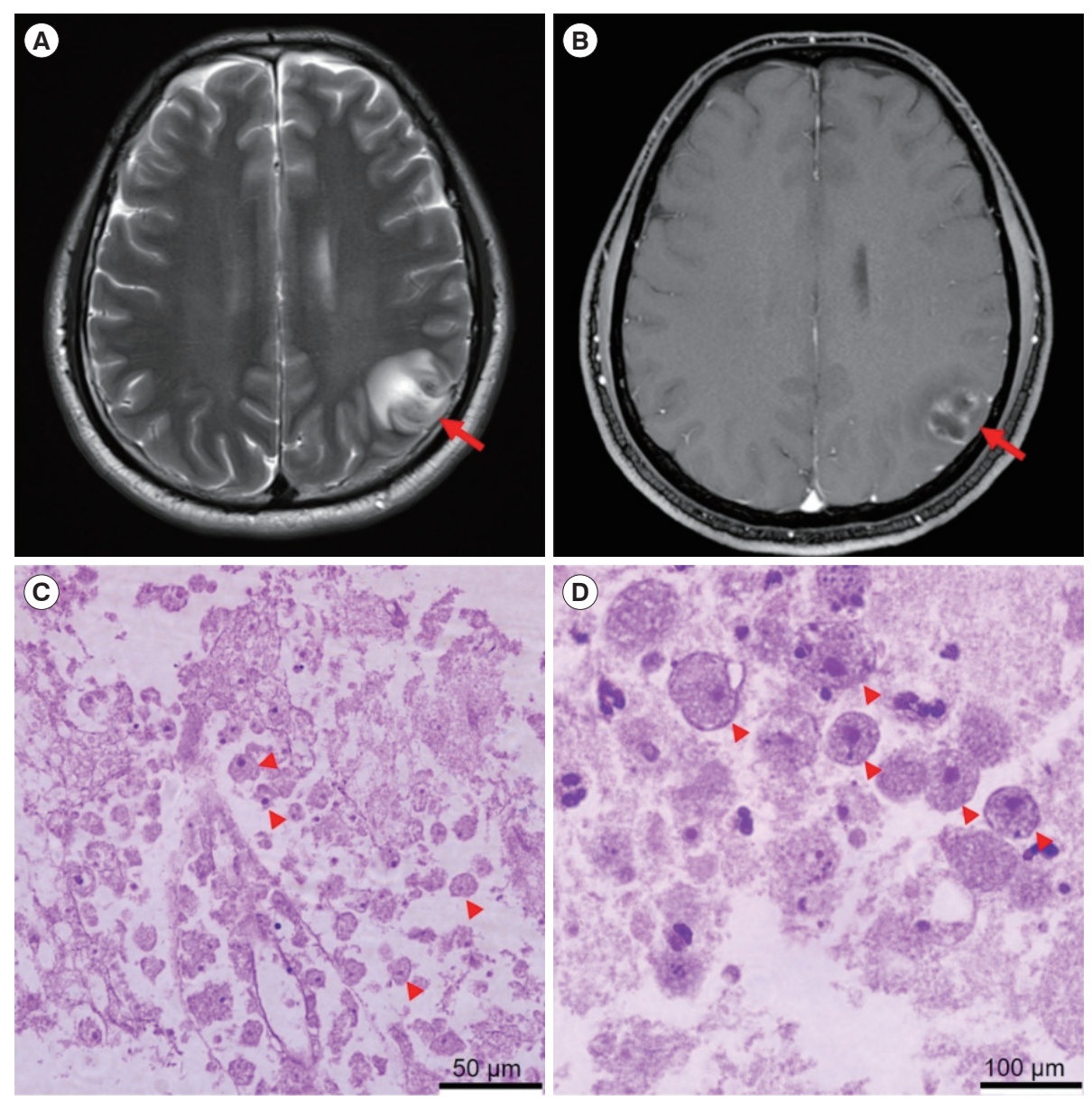

Fig. 1. Magnetic resonance image (MRI) and brain biopsy. T2-weighted MRI showing (A) a lesion $(\sim 21 \mathrm{~mm} \times 18 \mathrm{~mm})$ with an irregular, marginated, heterogeneous dark signal and (B) marginal thin-rim enhancement of gadolinium at the left parietal cortex with surrounding edema (red arrows). (C, D) Hematoxylin and eosin staining of brain biopsy shows numerous amoebic trophozoites (20-25 $\mu \mathrm{m})$ in the hemorrhagic necrosis background (arrowheads). Magnification: $\times 200$ (C), $\times 1,000$ (D). 
(Fig. 1A, B). An open biopsy of the brain performed immediately upon arrival suggested amebic infection in the brain with inflammatory cell infiltration and necrotizing vasculitis. Numerous amebic trophozoites (20-25 $\mu \mathrm{m}$ ) were identified by microscopy (Fig. 1C, D), and anti-Entamoeba histolytica and anti-Toxoplasma gondii IgM antibody titers were positive and negative, respectively. Laboratory findings were normal. An initial laboratory evaluation revealed a white blood cell count of $6.65 \times 10^{9} / \mathrm{L}$, hemoglobin of $151 \mathrm{~g} / \mathrm{L}$, serum sodium level of $138 \mathrm{mmol} / \mathrm{L}$, and serum glucose level of $7.05 \mathrm{mmol} / \mathrm{L}$. A diagnosis of intracranial amebiasis was made based on the pathological findings, and the patient was treated with metronidazole, amphotericin $B$, and sulfadiazine/pyrimethamine. However, drowsiness and progressive hemiparesis seven days after the open biopsy indicated a deteriorated neurological status. Emergency MRI showed multiple new lesions on the opposite site of the brain and increased intracranial pressure, which was reduced by decompressive craniectomy.

The study protocol was approved by the Institutional Review Board of Yonsei University Sever-ance Hospital, Seoul, Korea (approval No. 4-2020-0201), and the patient provided written in-formed consent.

\section{DNA extraction}

A paraffin block of the sample was prepared immediately after the biopsy (February 21, 2020 at Eulji University Hospital). Two weeks after the biopsy, the paraffin block was transferred to the laboratory at Yonsei University College of Medicine, and DNA extraction, TA cloning, and PCR were performed on the same day. DNA was extracted from paraffin-embedded brain biopsy samples using the Exgene Formalin-Fixed, Paraffin-Embedded Tissue DNA Kit (GeneAll, Seoul, Korea) in accordance with the manufacturer's instructions and stored at $4^{\circ} \mathrm{C}$ until analyses.

\section{TA cloning}

We amplified protozoan 18S rRNA gene sequences by PCR using two sets of primers targeting the V4-5 and V9 regions of $18 \mathrm{~S}$ rRNA, which are typically used in eukaryotic metagenomics as they amplify the 18S rRNA of nearly all types of eukaryotic species [3]. Thus, this procedure can discriminate the $18 \mathrm{~S}$ rRNA gene sequences of patient and protozoan DNA. The 18S rRNA gene V4-5 and V9 regions of any eukaryotic species in the sample were cloned using the TOPcloner TA Kit (Enzynomics, Daejeon, Korea), in accordance with the manufacturer's instructions with the following primers: V4-5 region, V4-1 forward 5'-GCGGTAATTCCAGCTC-3' and V4-4 reverse 5'-GCCMTTCCGTCAA-
TTCC-3'; v9 region, $1391 f$ 5'-GTACACACCGCCCGTC-3' and EukBr 5'-TGATCCTTCTGCAGGTTCACCTAC-3' [3, 4]. The reaction conditions were as follows: initial denaturation for 5 minutes at $95^{\circ} \mathrm{C}$; 34 amplification cycles comprising denaturation for 30 seconds at $95^{\circ} \mathrm{C}$, annealing for 30 seconds at $55^{\circ} \mathrm{C}$, and extension for 45 seconds at $72^{\circ} \mathrm{C}$; and final extension for 5 minutes at $72^{\circ} \mathrm{C}$. The following day, recombinant colonies were cultured overnight in Luria-Bertani broth with ampicillin, and the recombinant plasmids from the recombinant clones were extracted using the Exprep Plasmid SV Mini Kit (GeneAll, Seoul, Korea). Forty-one plasmids recombinant with 18S rRNA V4-5 regions and 63 plasmids recombinant with 18S rRNA V9 re-gions were sequenced at Bionics (Seoul, Korea) using the M13F(-20) primer. Homology was de-termined using sequences from the National Center for Biotechnology Information (NCBI) Basic Local Alignment Search Tool (BLAST, https://blast.ncbi.nlm.nih. gov/Blast.cgi).

\section{Phylogenetic analysis}

The DNA sequences were compared with nucleotide sequences available in GenBank (NCBI). A phylogenetic tree was constructed using Molecular Evolutionary Genetics Analysis version X (MEGA-X) software (The Pennsylvania State University, PA, USA) [5].

\section{Conventional PCR for specific protozoal infection}

Conventional PCR was performed to detect the protozoa Entamoeba histolytica, Toxoplasma gondii, Naegleria fowleri, and Acanthamoeba spp. based on previous studies [6-9]. N. fowlerispecific PCR was performed using the following primer set to amplify the amplicon of predicted size $183 \mathrm{bp}$ : Nae3-F (5'-CAAACACCGTTATGACAGGG-3') and Nae3-R (5'-CTGGTTTCCCTCACCTTACG-3') [6]. Entamoeba histolytica-specific nested PCR was per-formed using the following primer sets to amplify the amplicon of predicted size 270 bp: Fw1 (5'-GCAGAAAAAAATAATAATAAC-3') and Rv1 (5'-TTCATTTGTTTTACTTTCA-3') for the first PCR and Fw2 (5'-CGCCCGCCGCGCGGCCGCGGCCGGCCGGGGGCACGCGGCGGCAGAAAAAAA-TAATAATAAC-3’) and Rv2 (5'-TTCATTTGTTTTACTTTCA-3') for the subsequent PCR [7]. Toxoplasma gondii-specific PCR was performed using the following primer set to amplify the amplicon of predicted size 469 bp: Tg1 (5'-AAAAATGTGGGAATGAAAGAG-3') and Tg2 (5'ACGAATCAACGGAACTGTAAT-3’) [8]. Acanthamoeba-specific PCR was performed using the following primer set to amplify the amplicon of predicted size 463 bp: JDP1 (5'-GGCCCAGATCGTTTACCGTGAA-3') and JDP2 (5'-TCTCACAAGCTGCTAGGGGAGTCA-3') [9]. To confirm the TA cloning results, $B$. 
mandrillaris-specific PCR was performed using the following primer sets to generate products of pre-dicted sizes 1075, 230, and $170 \mathrm{bp}$, respectively: 5'Balspec16S (5'-CGCATGTATGAAGAAGACCA-3') and 3'Balspec16S (5'-TTACCTATATAATTGTCGATACCA-3'); 5'Balspec 16S (5'-CGCATGTATGAAAGAAGACCA-3') and Balspec 16Sr 610 (5'-CCCCTTTTTAACTCTAGTCATATAGT-3'); and BalaF1451 (5'-TAACCTGCTAAATAGTCATGCCAAT-3') and BalaR1621 (5'-CAAACTTCCCTCGGCTAATCA3) [10-12].

\section{RESULTS}

Four days after the brain biopsy, the protozoan species in the brain lesion was identified as B. mandrillaris by TA cloning. However, the patient had already succumbed to brain death and died four weeks later.

The pathogen-specific PCR results ruled out E. histolytica, $N$. fowleri, Acanthamoeba spp., and T. gondii infections based on the absence of positive bands (Fig. 2A). Simultaneous TA cloning was performed using general eukaryotic primers to detect unexpected protozoan infections because we did not consider B. mandrillaris infection.

The homology analysis using NCBI BLAST showed that the 18S V4-5 and V9 regions of the protozoan pathogen in the sample exhibited $99.2 \%$ and $100 \%$ nucleotide identity with those of B. mandrillaris KF874819 and KT175741 in GenBank, respectively (Table 1).

The DNA of the patient and $B$. mandrillaris was detected at different rates when cloning the 18S rRNA V4-5 or V9 variable region. This is likely related to the variation in the GC content
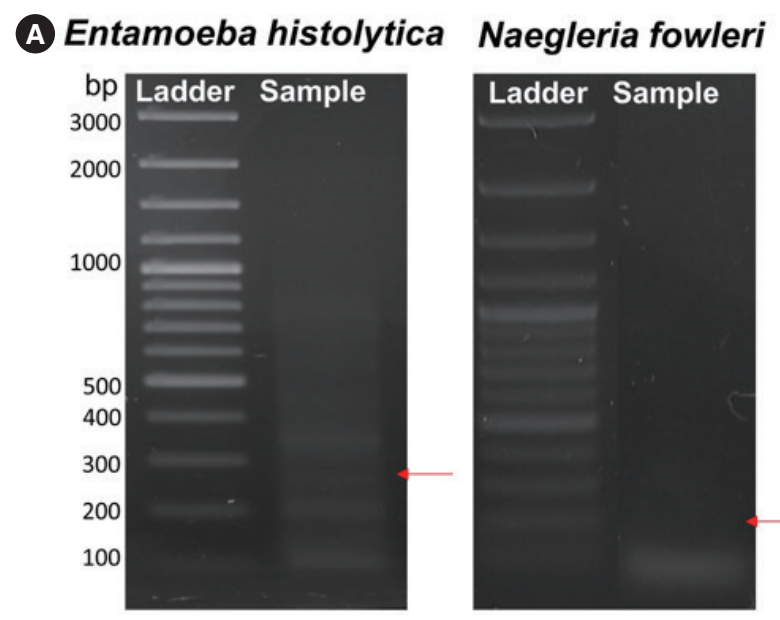

\section{B Balamuthia mandrillaris}

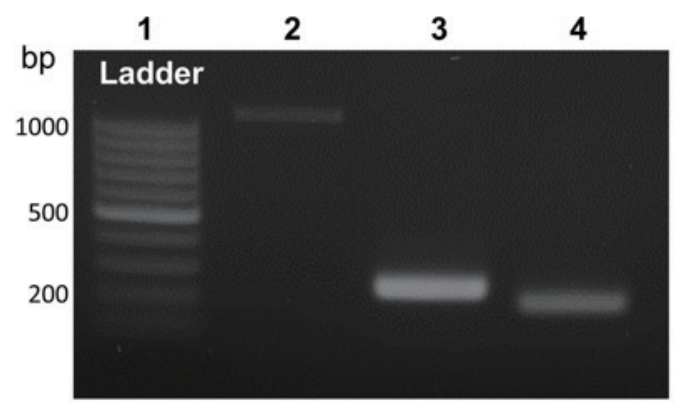

\section{Acanthamoeba spp. Toxoplasma gondii}
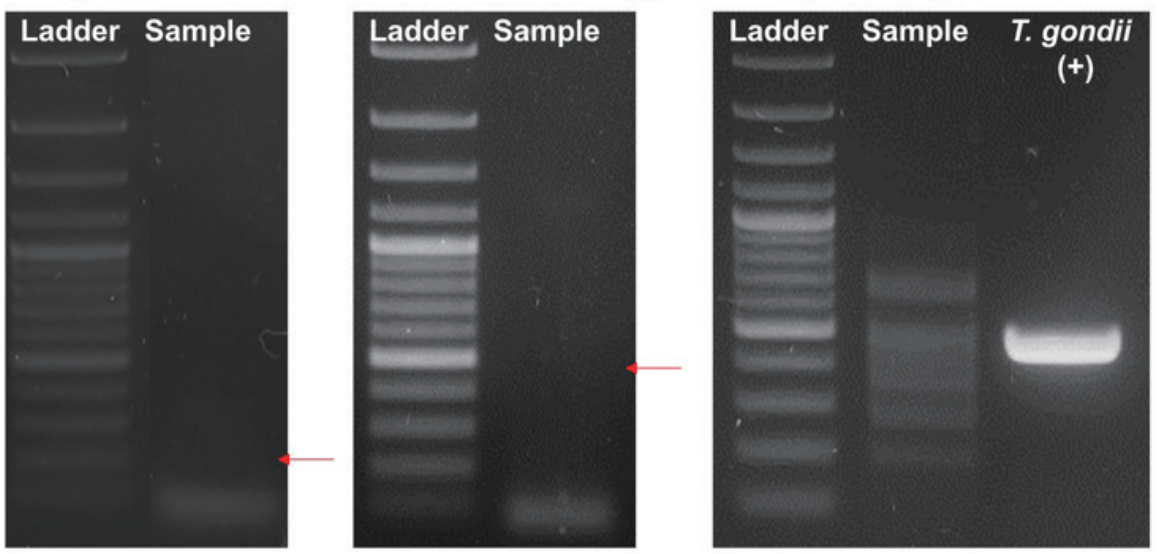

Fig. 2. Pathogen-specific PCR targeting (A) Entamoeba histolytica, Naegleria fowleri, Acanthamoeba spp., Toxoplasma gondii, and (B) Balamuthia mandrillaris. Red arrows show approximate band sizes of 270, 183, and 463 bp for E. histolytica, N. fowleri, and Acanthamoeba spp., respectively. Toxoplasma gondii DNA (469 bp) was used as the positive control. Balamuthia mandrillaris-specific PCR using the patient DNA was performed with three sets of primers. Lane 1: $100 \mathrm{bp}$ ladder. Lanes 2, 3, and 4: 5'Balspec 16S and 3'Balspec16S, 5'Balspec 16S and Balspec 16Sr 610, and BalaF1451 and BalaR1621 primers, respectively.

Table 1. Molecular identification (nucleotide identity) of the protozoa from the patient and previously published protozoan DNA sequences that required differential diagnoses

\begin{tabular}{|c|c|c|c|c|c|c|c|c|c|c|}
\hline \multirow{2}{*}{$\begin{array}{l}\text { 18S rRNA } \\
\text { region }\end{array}$} & \multicolumn{2}{|c|}{ Balamuthia mandrillaris } & \multicolumn{2}{|c|}{ Entamoeba histolytica } & \multicolumn{2}{|c|}{ Naegleria fowleri } & \multicolumn{2}{|c|}{ Acanthamoeba castellanii } & \multicolumn{2}{|c|}{ Toxoplasma gondii } \\
\hline & Identity & GenBank N & Identity & GenBank N & Identity & GenBank N & Identity & GenBank N & Identity & GenBank N \\
\hline V4-5 & $99.2 \%$ & KF874819 & $53.5 \%$ & AB426549 & $57.2 \%$ & U80059 & $70.3 \%$ & U07401 & $69.8 \%$ & L24381 \\
\hline V9 & $100 \%$ & KT175741 & $38.3 \%$ & AB426549 & $44.0 \%$ & U80059 & $35.7 \%$ & U07401 & $44.8 \%$ & L24381 \\
\hline
\end{tabular}


Table 2. Number of recombinant colonies containing 18S rRNA genes (V4-5 and V9 regions) of Balamuthia mandrillaris and Homo sapiens and their GC content and DNA length

\begin{tabular}{|c|c|c|c|c|c|c|}
\hline & $18 \mathrm{~S}$ & rRNA V4-5 region & & & rRNA V9 region & \\
\hline & Recombinant colonies (N) & GC content (\%) & DNA length (bp) & Recombinant colonies (N) & GC content (\%) & DNA length (bp) \\
\hline B. mandrillaris & 2 & 46.1 & 662 & 35 & 40.8 & 179 \\
\hline H. sapiens & 32 & 55.2 & 591 & 8 & 58.6 & 174 \\
\hline
\end{tabular}

*The total number of recombinant colonies examined was 41 and 63 for the V4-5 and V9 regions, respectively. Among them, 7 and 20 plasmids were found to be empty vectors.
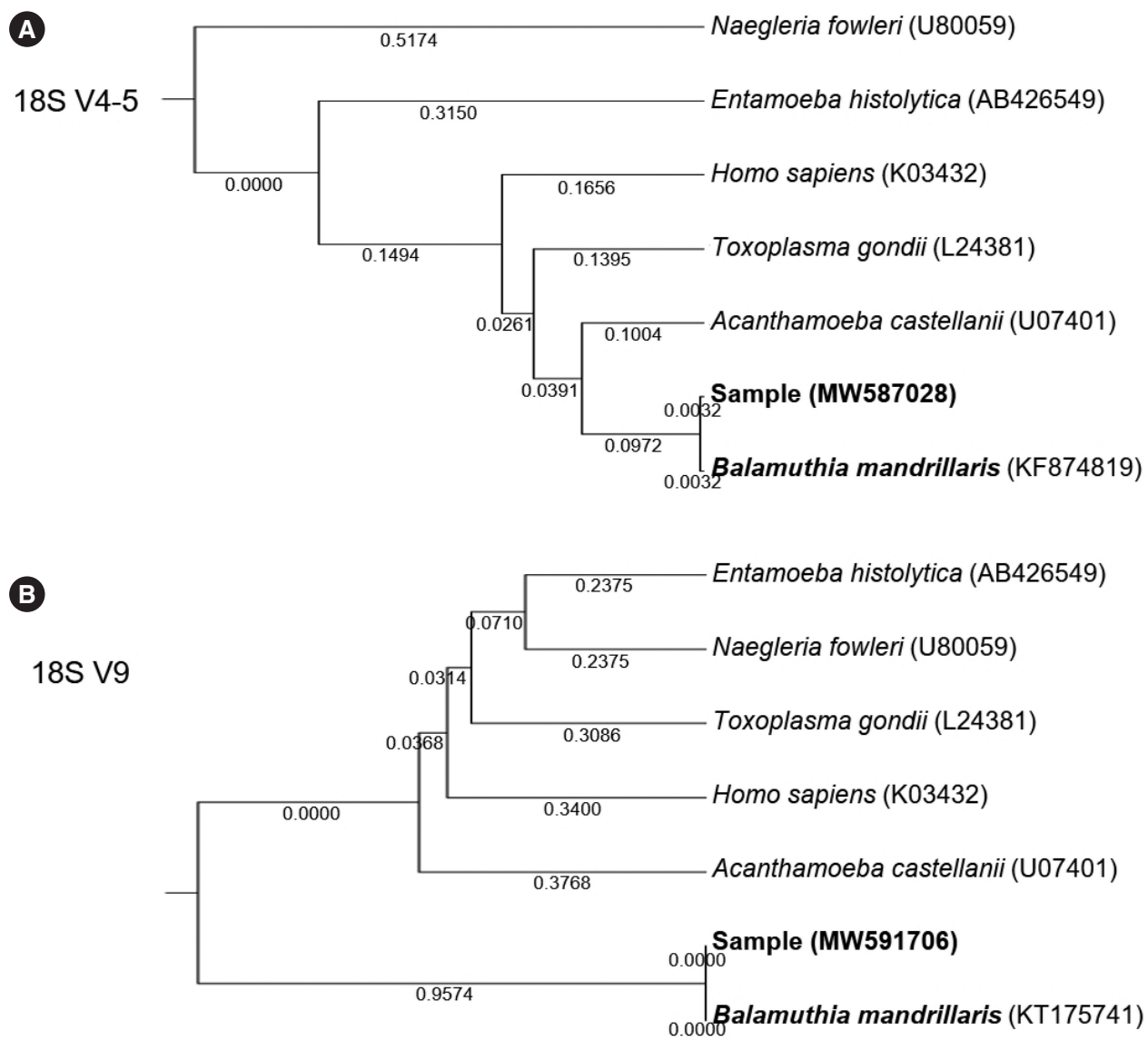

Fig. 3. Phylogenetic trees of the sequenced sample based on the (A) V4-5 region and (B) V9 region of the 18S rRNA gene. The total branch lengths of phylogenetic trees for the $\mathrm{V} 4-5$ and $\mathrm{V} 9$ regions were 0.8465 and 2.2305 , respectively, computed using the maximum composite likelihood method and measured in base substitutions per site. Sequences of 18S rRNA genes of reference organisms were obtained from GenBank (National Center for Biotechnology Information).

and DNA sequence lengths in the two genes (Table 2).

B. mandrillaris infection was reconfirmed using B. mandrillaris-specific PCR with three primer sets. The bands resolved at the expected points in gel electrophoresis and their DNA sequences were identical to published $B$. mandrillaris nucleotide sequences (Fig. 2B).

The phylogenetic tree of 18S rRNA V4-5 and V9 sequences showed that the sequence of the sample was distant from those of other protozoan candidates, including E. histolytica, N. fowleri, Acanthamoeba castellanii, and T. gondii (Fig. 3).

\section{DISCUSSION}

Naegleria fowleri, Acanthamoeba spp., and B. mandrillaris are established etiological agents of encephalitis. $N$. fowleri causes primary amebic meningoencephalitis with a fulminant disease 
course, and Acanthamoeba spp. and B. mandrillaris cause granulomatous amebic encephalitis [13, 14]. Among approximately 150 patients with Balamuthia amebic encephalitis reported worldwide, only 10 have survived [15]. B. mandrillaris enters the host via skin wounds or inhalation and spreads through the bloodstream to the central nervous system, resulting in fever, seizures, cranial nerve dysfunction, motor deficits, increased intracranial pressure, and altered consciousness [16, 17]. The survival rate of patients with $B$. mandrillaris encephalitis can be $<2 \%$ or a maximum of approximately $6.7 \%$ due to its rarity, difficulty in diagnosis, and lack of effective treatment protocols [16, 18]. The US Center for Disease Control and Prevention recommends pentamidine, sulfadiazine, flucytosine, fluconazole, clarithromycin, azithromycin, and miltefosine to treat B. mandrillaris encephalitis [19-23].

To the best of our knowledge, this is the first case of B. mandrillaris encephalitis in Korea identified using a molecular (TA cloning) approach. Although a 71-year-old man in Korea was diagnosed as having B. mandrillaris encephalitis in 2019 based on microscopy findings [24], including the detection of trophozoites and cysts of $B$. mandrillaris as well as areas of granuloma in host tissues, this protozoan is morphologically indistinguishable from Acanthamoeba spp. [13, 15, 25]. Balamuthia culture requires feeder cells and several weeks for cell proliferation. $A$ diagnosis should be confirmed by PCR-based detection of $B$. mandrillaris DNA in tissues [26]. However, this technique is not widely available given the extreme rarity of $B$. mandrillaris encephalitis [1].

In the present case, pathogen-specific PCR was negative for E. histolytica, N. fowleri, Acanthamoeba spp., and T. gondii. T. gondii was the only positive control because protozoal and infected tissue samples were not available in our laboratory. This is likely a common situation in other research institutions, as it is difficult to maintain positive samples for all possible infectious diseases.

We applied TA cloning in this case, because it can provide results faster than metagenomic NGS and the protozoan cells in the brain tissues detected by microscopy were enough to enable confirmation using TA cloning by separating amplified protozoan and patient $18 \mathrm{~S}$ rRNA genes. With this approach, we identified B. mandrillaris genes in the brain biopsy sample four days after receiving the paraffin-embedded sample. Theoretically, TA cloning can generate results within 48 hours; therefore, it should be among the diagnostic choices available to detect infectious agents of unknown etiology in clinical practice.

Cloning with the primer amplifying the 18S rRNA V9 region resulted in 35 colonies with the $B$. mandrillaris gene and eight colonies with the patient gene. In contrast, cloning with the $18 \mathrm{~S}$ V4-5 primer resulted in two and 32 colonies with $B$. mandrillaris and human genes, respectively (Table 2). This difference is probably due to the length and the GC content of the PCR products (Table 2). The PCR product of the 18S V4-5 region of $B$. mandrillaris was $11 \%$ longer than that of the human gene. Furthermore, the GC content of $B$. mandrillaris PCR products increased from $40.8 \%$ for the V9 primer to $46.1 \%$ for the V4-5 primer, which might have reduced PCR efficiency. Various primers can be used to amplify different $18 \mathrm{~S}$ rRNA regions during TA cloning to increase the probability of protozoa detection.

This study is limited by the fact that we identified B. mandrillaris by TA cloning only in a brain lesion. As brain tissue biopsies can cause neurological disorders, diagnosing $B$. mandrillaris infection in samples other than brain tissues such as the blood and cerebrospinal fluid might be more helpful for patients undergoing clinical treatment.

In conclusion, to the best of our knowledge, this is the first study to describe the molecular identification of $B$. mandrillaris encephalitis in Korea using TA cloning. This method should be considered for detecting infectious agents of unknown etiology in routine clinical practice.

\section{ACKNOWLEDGMENTS}

None declared.

\section{AUTHOR CONTRIBUTIONS}

Kim JY and Kim SM contributed to the conception and design of the study; Yoo HD and Yeom JS were involved in clinical evaluation; Yi MH and Kim M interpreted the results; Kim JY drafted the manuscript; and Yong TS supervised the study. All authors read and approved the final manuscript.

\section{CONFLICT OF INTEREST}

None declared.

\section{RESEARCH FUNDING}

This work was supported by the National Research Foundation of Korea (NRF) grants funded by the Korean Government (Ministry of Education, Science and Technology; grant numbers 2019R1A2B5B01069843 and 2020R1I1A2074562). 


\section{ORCID}

Ju Yeong Kim

Myung-Hee Yi

Myungjun Kim

Joon-Sup Yeom

Hyun Dong Yoo

Seong Min Kim

Tai-Soon Yong https://orcid.org/0000-0003-2456-6298 https://orcid.org/0000-0001-9537-5726

https://orcid.org/0000-0001-9308-4917

https://orcid.org/0000-0001-8940-7170

https://orcid.org/0000-0003-1381-2000 https://orcid.org/ 0000-0001-7161-0226

https://orcid.org/0000-0002-3445-0769

\section{REFERENCES}

1. Wilson MR, Shanbhag NM, Reid MJ, Singhal NS, Gelfand JM, Sample HA, et al. Diagnos-ing Balamuthia mandrillaris encephalitis with metagenomic deep sequencing. Ann Neurol 2015;78:722-30.

2. Wilson MR, Naccache SN, Samayoa E, Biagtan M, Bashir H, Yu G, et al. Actionable diagnosis of neuroleptospirosis by next-generation sequencing. N Engl J Med 2014;370:2408-17.

3. Popovic A, Bourdon C, Wang PW, Guttman DS, Voskuijl W, Grigg ME, et al. Design and application of a novel two-amplicon approach for defining eukaryotic microbiota. Microbiome 2018;6:228.

4. Gilbert JA, Jansson JK, Knight R. The Earth Microbiome project: successes and aspirations. BMC Biol 2014;12:69.

5. Kumar S, Stecher G, Li M, Knyaz C, Tamura K. MEGA X: Molecular Evolutionary Genetics Analysis across computing platforms. Mol Biol Evol 2018;35:1547-9

6. Kang H, Seong GS, Sohn HJ, Kim JH, Lee SE, Park MY, et al. Effective PCR-based detection of Naegleria fowleri from cultured sample and PAM-developed mouse. Eur J Protistol 2015;51:401-8.

7. López-López P, Martínez-López MC, Boldo-León XM, Hernández-Díaz Y, González-Castro TB, Tovilla-Zárate CA, et al. Detection and differentiation of Entamoeba histolytica and Entamoeba dispar in clinical samples through PCR-denaturing gradient gel electrophoresis. Braz J Med Biol Res 2017;50:e5997.

8. Lukášová R, Kobédová K, Halajian A, Bártová E, Murat JB, Rampedi KM, et al. Molecular detection of Toxoplasma gondii and Neospora caninum in birds from South Africa. Acta Trop 2018;178:93-6.

9. Pasricha G, Sharma S, Garg P, Aggarwal RK. Use of 18 S rRNA genebased PCR assay for diagnosis of Acanthamoeba keratitis in non-contact lens wearers in India. J Clin Microbiol 2003;41:3206-11.

10. Booton GC, Carmichael JR, Visvesvara GS, Byers TJ, Fuerst PA. Identification of Balamuthia mandrillaris by PCR assay using the mitochondrial 16S rRNA gene as a target. J Clin Microbiol 2003;41:453-5.

11. Yamanouchi K, Arima H, Sakamoto $\mathrm{Y}$, Kanto K, Kasai K, Ito K, et al.
First report of the isola-tion of Balamuthia mandrillaris in the northern region of Japan. Parasitol Res 2018;117:2895-900.

12. Wu X, Yan G, Han S, Ye Y, Cheng X, Gong H, et al. Diagnosing Balamuthia mandrillaris encephalitis via next-generation sequencing in a 13-year-old girl. Emerg Microbes Infect 2020;9:1379-87.

13. da Rocha-Azevedo B, Tanowitz HB, Marciano-Cabral F. Diagnosis of infections caused by pathogenic free-living amoebae. Interdiscip Perspect Infect Dis 2009;2009:251406.

14. Visvesvara GS. Free-living amebae as opportunistic agents of human disease. J Neuroparasitology 2010;1:1-13.

15. Krasaelap A, Prechawit S, Chansaenroj J, Punyahotra P, Puthanakit T, Chomtho K, et al. Fatal Balamuthia amebic encephalitis in a healthy child: A case report with review of survival cases. Korean J Parasitol 2013;51:335-41.

16. Diaz JH. The public health threat from Balamuthia mandrillaris in the southern United States. J La State Med Soc 2011;163:197-204.

17. Schuster FL and Visvesvara GS. Free-living amoebae as opportunistic and non-opportunistic pathogens of humans and animals. Int J Parasitol 2004;34:1001-27.

18. Matin A, Siddiqui R, Jayasekera S, Khan NA. Increasing importance of Balamuthia mandrillaris. Clin Microbiol Rev 2008;21:435-48.

19. Cary LC, Maul E, Potter C, Wong P, Nelson PT, Given C, et al. Balamuthia mandrillaris meningoencephalitis: survival of a pediatric patient. Pediatrics 2010;125:e699-703.

20. Deetz TR, Sawyer MH, Billman G, Schuster FL, Visvesvara GS. Successful treatment of Balamuthia amoebic encephalitis: presentation of 2 cases. Clin Infect Dis 2003;37:1304-12.

21. Jung S, Schelper RL, Visvesvara GS, Chang HT. Balamuthia mandrillaris meningoencephalitis in an immunocompetent patient: an unusual clinical course and a favorable outcome. Arch Pathol Lab Med 2004;128: 466-8.

22. Martínez DY, Seas C, Bravo F, Legua P, Ramos C, Cabello AM, et al. Successful treatment of Balamuthia mandrillaris amoebic infection with extensive neurological and cutaneous involvement. Clin Infect Dis 2010; 51:e7-11.

23. Orozco LD, Khan MA, Fratkin JD, Hanigan WC. Asymptomatic aneurysm of the cavernous and supraclinoid internal carotid artery in a patient with Balamuthia mandrillaris encephalitis. J Clin Neurosci 2011;18: 1118-20.

24. Kum SJ, Lee HW, Jung HR, Choe M, Kim SP. Amoebic encephalitis caused by Balamuthia mandrillaris. J Pathol Transl Med 2019;53: 327-31.

25. Guarner J, Bartlett J, Shieh WJ, Paddock CD, Visvesvara GS, Zaki SR. Histopathologic spectrum and immunohistochemical diagnosis of amebic meningoencephalitis. Mod Pathol 2007;20:1230-7.

26. Schuster FL, Yagi S, Gavali S, Michelson D, Raghavan R, Blomquist I, et al. Under the radar: Balamuthia amebic encephalitis. Clin Infect Dis 2009;48:879-87. 